\title{
Platinum Participation in the Hydrogenation of Phenylacetylene by $\mathrm{Ru}_{5}(\mathrm{CO})_{15}(\mathrm{C})\left[\mathbf{P t}\left(\mathrm{PBu}_{3}^{\mathrm{t}}\right)\right]$
}

\author{
Richard D. Adams*, Burjor Captain and Lei Zhu \\ Department of Chemistry and Biochemistry, \\ University of South Carolina Columbia, SC 29208.
}

\section{Experimental Section}

\section{General Data}

All the reactions were performed under a nitrogen atmosphere using Schlenk techniques. Reagent grade solvents were dried by the standard procedures and were freshly distilled prior to use. Infrared spectra were recorded on an AVATAR 360 FT-IR spectrophotometer. ${ }^{1} \mathrm{H}$ NMR and ${ }^{31} \mathrm{P}$ NMR were recorded on a Varian Inova 400 spectrometer operating at 399 and $162 \mathrm{MHz}$, respectively. ${ }^{31} \mathrm{P}$ NMR spectra were externally referenced against $85 \%$ ortho $-\mathrm{H}_{3} \mathrm{PO}_{4}$. Elemental analyses were performed by Desert Analytics (Tucson, AZ). $\mathrm{Pt}\left(\mathrm{PBu}_{3}^{\mathrm{t}}\right)_{2}$ was purchased from Strem, and phenylacetylene and trimethylamine oxide were purchased from Aldrich and were used without further purification. $\mathrm{Ru}_{5}(\mathrm{CO})_{15}(\mathrm{C})\left[\mathrm{PtPBu}_{3}^{\mathrm{t}}\right], \mathbf{1},{ }^{1 \mathrm{a},}{ }^{1 \mathrm{~b}}$ was prepared according to the published procedures. Product separations were performed by TLC in air on Analtech 0.25 and $0.5 \mathrm{~mm}$ silica gel $60 \AA_{\mathrm{F}_{254}}$ glass plates.

\section{Preparation of $\mathrm{Ru}_{5}(\mathrm{CO})_{14}\left(\mu_{6^{-}} \mathrm{C}\right)\left[\mathrm{PtPBu}_{3}^{\mathrm{t}}\right](\mu-\mathrm{H})_{2}, 2$.}

A $9.3 \mathrm{mg}$ amount of $1(0.0070 \mathrm{mmol})$ was dissolved in $10 \mathrm{~mL}$ heptane in a $25 \mathrm{~mL}$ three-neck flask. The solution was then heated to reflux and purged with hydrogen (1 atm) for $1.5 \mathrm{~h}$. The solvent was then removed in vacuo and the product was separated by TLC by using a 4:1 hexane-methylene chloride solvent mixture to yield $6.3 \mathrm{mg}(68 \%)$ of 
a dark gray $\mathrm{Ru}_{5}(\mathrm{CO})_{14}\left(\mu_{6}-\mathrm{C}\right)\left[\mathrm{PtPBu}_{3}^{\mathrm{t}}\right](\mu-\mathrm{H})_{2}$, 2. Spectral data for 2: IR $v_{\mathrm{CO}}\left(\mathrm{cm}^{-1}\right.$ in $\mathrm{CH}_{2} \mathrm{Cl}_{2}$ ): 2087 (m), 2052 (s), 2015 (s), 1971 (w, sh), 1821 (w, br). ${ }^{1} \mathrm{H}$ NMR (in toluene$\left.\mathrm{d}_{8}\right): \delta=1.22\left(\mathrm{~d}, 27 \mathrm{H}, \mathrm{CH}_{3},{ }^{3} \mathrm{~J}_{\mathrm{P}-\mathrm{H}}=13 \mathrm{~Hz}\right),-13.86\left(\mathrm{~d}, 2 \mathrm{H}\right.$, hydride, ${ }^{1} \mathrm{~J}_{\mathrm{Pt}-\mathrm{H}}=774 \mathrm{~Hz},{ }^{2} \mathrm{~J}_{\mathrm{P}-\mathrm{H}}=$ $8 \mathrm{~Hz}$ ). ${ }^{31} \mathrm{P}\left\{{ }^{1} \mathrm{H}\right\}$ NMR (in toluene- $\left.\mathrm{d}_{8}\right): \delta=85.3\left(\mathrm{~s}, 1 \mathrm{P},{ }^{1} \mathrm{~J}_{\mathrm{Pt}-\mathrm{P}}=4739 \mathrm{~Hz}\right.$ ). Anal. Calc.: $\mathrm{C}$, 24.77; H, 2.22. Found: C, 25.13; H, $2.50 \%$.

\section{Preparation of $\mathrm{Ru}_{5}(\mathrm{CO})_{13}\left(\mu_{5}-\mathrm{C}\right)\left(\mathrm{PhC}_{2} \mathrm{H}\right)\left[\mathrm{PtPBu}_{3}{ }_{3}\right], 3$.}

A $16 \mathrm{mg}$ amount of $1(0.012 \mathrm{mmol})$ was dissolved in $20 \mathrm{~mL} \mathrm{CH}_{2} \mathrm{Cl}_{2}$ in a $50 \mathrm{~mL}$ three-neck flask. Phenylacetylene $(0.010 \mathrm{~mL}, 0.090 \mathrm{mmol})$ was added to the reaction, which was then heated to reflux for $1 \mathrm{~h}$. After cooling, the solvent was removed in vacuo, and the product was purified by TLC by using 6:1 hexane-methylene chloride solvent mixture to yield $6.8 \mathrm{mg}(41 \%)$ of a $\mathrm{Ru}_{5}(\mathrm{CO})_{13}\left(\mu_{5}-\mathrm{C}\right)\left(\mathrm{PhC}_{2} \mathrm{H}\right)\left[\mathrm{PtPBu}_{3}^{\mathrm{t}}\right], \mathbf{3}$. Spectral data for 3: IR $v_{\mathrm{CO}}\left(\mathrm{cm}^{-1}\right.$ in $\left.\mathrm{CH}_{2} \mathrm{Cl}_{2}\right)$ : 2076 (vs), 2044 (vs), 2027 (s), 2014 (vs), 1985 (w, sh), 1955 (vw, sh). ${ }^{1} \mathrm{H}$ NMR (in $\left.\mathrm{CDCl}_{3}\right): \delta=8.58\left(\mathrm{~d}, 1 \mathrm{H}, \mathrm{CH},{ }^{3} \mathrm{~J}_{\mathrm{P}-\mathrm{H}}=16 \mathrm{~Hz}\right.$ ), $7.3-7.4(\mathrm{~m}, 5 \mathrm{H}, \mathrm{Ph}), 1.50\left(\mathrm{~d}, 27 \mathrm{H}, \mathrm{CH}_{3},{ }^{3} \mathrm{~J}_{\mathrm{P}-\mathrm{H}}=13 \mathrm{~Hz}\right) .{ }^{31} \mathrm{P}\left\{{ }^{1} \mathrm{H}\right\} \mathrm{NMR}$ (in $\left.\mathrm{CDCl}_{3}\right): \delta=$ 113.4 (s, 1P, ${ }^{1} \mathrm{~J}_{\mathrm{Pt}-\mathrm{P}}=4545 \mathrm{~Hz}$ ). Anal. Calc.: C, 29.57; H, 2.39. Found: C, 28.47; H, $2.15 \%$.

\section{Preparation of $\operatorname{Ru}_{5}(\mathrm{CO})_{12}\left(\mu_{5}-\mathrm{C}\right)\left[\mathrm{PtPBu}_{3}^{\mathrm{t}}\right]\left(\mathrm{PhC}_{2} \mathrm{H}\right)(\mu-\mathrm{H})_{2}, 4$.}

A $22.5 \mathrm{mg}$ amount of $\mathbf{3}(0.016 \mathrm{mmol})$ was dissolved in $25 \mathrm{~mL} \mathrm{CH}_{2} \mathrm{Cl}_{2}$. Through this solution $\mathrm{H}_{2}$ was purged at $1 \mathrm{~atm} .5 .4 \mathrm{mg}$ of $\mathrm{Me}_{3} \mathrm{NO} 2 \mathrm{H}_{2} \mathrm{O}(0.049 \mathrm{mmol})$ was then added to this solution and stirred at reflux for $1 \mathrm{~h}$. The solvent was then removed in vacuo and the product was separated by TLC by using a 4:1 hexane-methylene chloride solvent mixture to yield $4.2 \mathrm{mg}(19 \%)$ of a dark gray $\operatorname{Ru}_{5}(\mathrm{CO})_{12}\left(\mu_{5^{-}}\right.$ 
C) $\left[\mathrm{PtPBu}_{3}^{\mathrm{t}}\right]\left(\mathrm{PhC}_{2} \mathrm{H}\right)(\mu-\mathrm{H})_{2}$, 4. IR $v_{\mathrm{CO}}\left(\mathrm{cm}^{-1}\right.$ in $\left.\mathrm{CH}_{2} \mathrm{Cl}_{2}\right): 2083$ (s), 2047 (s), 2023 (vs), 2004 (w, sh), 1977 (w, sh), 1948 (vw, sh). ${ }^{1} \mathrm{H}$ NMR (in toluene-d $\mathrm{d}_{8}$ ): $\delta=9.76$ (dd, 1H, CH, $\left.{ }^{3} \mathrm{~J}_{\mathrm{H}-\mathrm{H}}=1.5 \mathrm{~Hz},{ }^{3} \mathrm{~J}_{\mathrm{P}-\mathrm{H}}=4 \mathrm{~Hz},{ }^{2} \mathrm{~J}_{\mathrm{Pt}-\mathrm{H}}=42 \mathrm{~Hz}\right), 7.72(\mathrm{~d}, 2 \mathrm{H}, \mathrm{Ph}-\mathrm{H}), 7.18(\mathrm{t}, 2 \mathrm{H}, \mathrm{Ph}-\mathrm{H}), 7.05$ $(\mathrm{t}, 1 \mathrm{H}, \mathrm{Ph}-\mathrm{H}), 0.99\left(\mathrm{~d}, 27 \mathrm{H}, \mathrm{CH}_{3},{ }^{3} \mathrm{~J}_{\mathrm{P}-\mathrm{H}}=13 \mathrm{~Hz}\right), \quad-9.56$ (ddd observed as a dt, $1 \mathrm{H}$, hydride on Pt, $\left.{ }^{2} \mathrm{~J}_{\mathrm{P}-\mathrm{H}}=1.5 \mathrm{~Hz},{ }^{2} \mathrm{~J}_{\mathrm{H}-\mathrm{H}}=4 \mathrm{~Hz},{ }^{3} \mathrm{~J}_{\mathrm{H}-\mathrm{H}}=1.5 \mathrm{~Hz},{ }^{1} \mathrm{~J}_{\mathrm{Pt}-\mathrm{H}}=641 \mathrm{~Hz}\right),-18.55(\mathrm{~d}$, hydride on Ru, ${ }^{2} \mathrm{~J}_{\mathrm{H}-\mathrm{H}}=4 \mathrm{~Hz}$ ). ${ }^{31} \mathrm{P}\left\{{ }^{1} \mathrm{H}\right\}$ NMR (in toluene- $\left.\mathrm{d}_{8}\right): \delta=124.0\left(\mathrm{~s}, 1 \mathrm{P},{ }^{1} \mathrm{~J}_{\mathrm{Pt}-\mathrm{P}}=3839\right.$ Hz). Anal. Calc.: C, 29.27; H, 2.61. Found: C, 29.63; H, $2.44 \%$.

\section{General Procedures for the Catalysis Studies.}

Phenylacetylene and $d_{8}$-toluene were purchased from Aldrich and were used without further purification. Turnover rates were obtained by measuring the amount of product formed by integrating the signals by ${ }^{1} \mathrm{H}$ NMR spectroscopy.

Catalytic Hydrogenation of Phenylacetylene.

1) In a typical run, a $5.3 \mathrm{mg}$ amount of $3(0.00384 \mathrm{mmol}), 2.1 \mu \mathrm{L}$ of $\mathrm{PhC}_{2} \mathrm{H}$ (0.192 mmol, 50 equivalents), were dissolved in $2 \mathrm{~mL}$ of $d_{8}$-toluene in a stainless steel Parr pressure reactor. The reactor was pressurized with $30 \mathrm{psi}$ of $\mathrm{H}_{2}$, placed in an oil bath maintained at $80{ }^{\circ} \mathrm{C}$, and allowed to stir for $1 \mathrm{~h}$. Analysis by ${ }^{1} \mathrm{H}$ NMR spectroscopy showed that $40 \%$ of the $\mathrm{PhC}_{2} \mathrm{H}$ was converted to styrene (20 turnovers).

2) Similarly $5.2 \mathrm{mg}$ of $4(0.00384 \mathrm{mmol})$ converted $41 \%$ of $\mathrm{PhC}_{2} \mathrm{H}$ to styrene (21 turnovers).

3) $4.2 \mathrm{mg}$ of $\mathrm{Ru}_{6}(\mathrm{CO})_{17}\left(\mu_{6}-\mathrm{C}\right)^{2}(0.00384 \mathrm{mmol})$ was treated with $\mathrm{PhC}_{2} \mathrm{H}$ and hydrogen under the same conditions described above. No styrene was detected by ${ }^{1} \mathrm{H}$ NMR spectroscopy. 


\section{Crystallographic Analysis.}

Dark red single crystals of $\mathbf{2}$ suitable for diffraction analysis were grown by slow evaporation of solvent from a diethyl ether solution at $-20^{\circ} \mathrm{C}$. Dark red crystals of $\mathbf{3}$ and 4 suitable for diffraction analysis were grown by slow evaporation of solvent from a benzene/octane solution at $8{ }^{\circ} \mathrm{C}$. Each data crystal was glued onto the end of thin glass fiber. X-ray intensity data were measured using a Bruker SMART APEX CCD-based diffractometer using Mo K $\alpha$ radiation $(\lambda=0.71073 \AA)$. The raw data frames were integrated with the SAINT+ program by using a narrow-frame integration algorithm. ${ }^{3}$ Correction for the Lorentz and polarization effects were also applied by SAINT. An empirical absorption correction based on the multiple measurement of equivalent reflections was applied by using the program SADABS. All three structures were solved by a combination of direct methods and difference Fourier syntheses, and refined by fullmatrix least-squares on $\mathrm{F}^{2}$, by using the SHELXTL software package. ${ }^{4}$ Crystal data, data collection parameters, and results of the analyses for compounds $\mathbf{2}-\mathbf{4}$ are listed in Table 1.

Compound 2 crystallized in the orthorhombic crystal system. The space group Pccn was identified on the basis of the systematic absences in the intensity data. All nonhydrogen atoms were refined with anisotropic displacement parameters. Both hydride ligands were located and refined with isotropic displacement parameters. A molecule of $\mathrm{C}_{2} \mathrm{H}_{5} \mathrm{OC}_{2} \mathrm{H}_{5}$ from the crystallization solvent cocrystallized with the complex. The solvent was included in the analysis and was satisfactorily refined with anisotropic 
thermal parameters. Hydrogen atoms were placed in geometrically idealized positions and included as standard riding atoms.

Compounds $\mathbf{3}$ and $\mathbf{4}$ crystallized in the triclinic crystal system. The space group $P-1$ was assumed and confirmed by the successful solution and refinement of the structure. All nonhydrogen atoms were refined with anisotropic displacement parameters. The hydrogen atom on the phenylacetylene ligand in both compounds were located and refined with isotropic displacement parameters. The hydride ligands in $\mathbf{4}$ were located and refined with isotropic displacement parameters. Compound 3 cocrystallized with half a molecule of octane from the crystallization solvent. The octane molecule was refined with isotropic displacement parameters. Five geometric restraints were used in modeling the octane molecule, which was disordered about an inversion center. Compound 4 co-crystallized with half a molecule of octane and half a molecule of benzene from the crystallization solvent. The solvent molecules were refined with anisotropic displacement parameters. Hydrogen atoms were placed in geometrically idealized positions and refined as standard riding atoms. 


\section{Reference:}

1. (a) Adams, R. D.; Captain, B.; Fu, W.; Pellechia, P. J.; Smith, M. D. Angew.

Chem. Int. Ed. 2002, 41, 1951. (b) Adams, R. D.; Captain, B.; Fu, W.; Pellechia, P. J.; Smith, M. D. Inorg. Chem., 2003, 42, 2094.

2. Nicholls, J. N.; Vargas, M. D.; Hriljac, J.; Sailor, M. Inorg. Synth. 1989, 26, 283.

3. SAINT+ Version 6.2a. Bruker Analytical X-ray System, Inc., Madison, Wisconsin, USA, 2001.

4. G. M. Sheldrick, SHELXTL Version 6.1; Bruker Analytical X-ray Systems, Inc., Madison, Wisconsin, USA, 1997. 


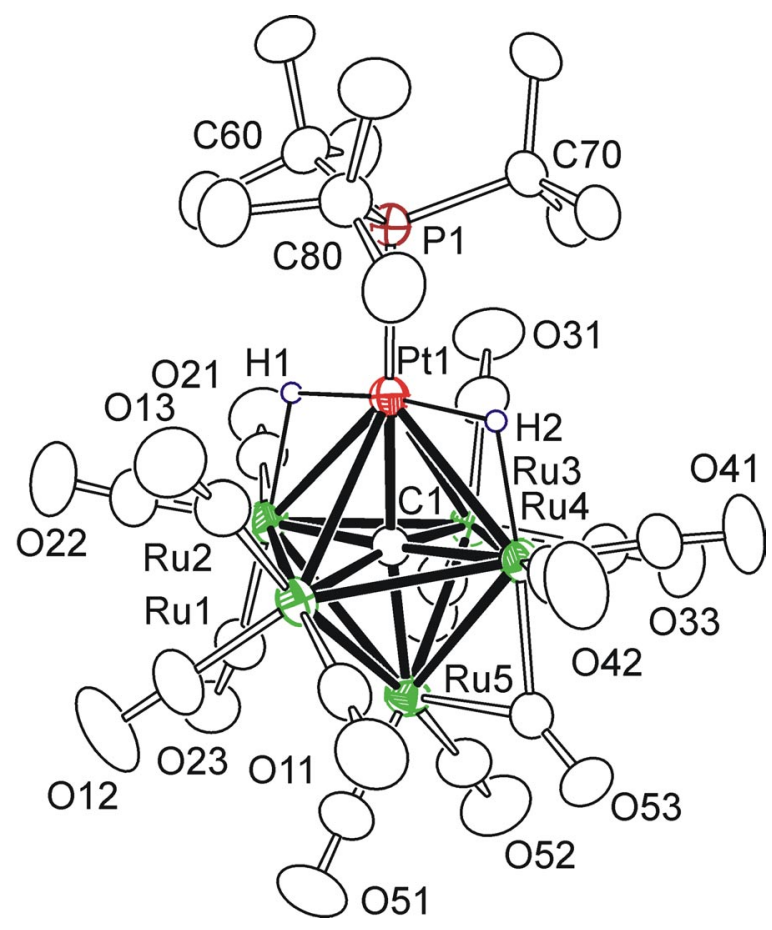

Figure 1. An ORTEP diagram of $\mathrm{Ru}_{5}(\mathrm{CO})_{14}\left(\mu_{6}-\mathrm{C}\right)\left[\mathrm{Pt}\left(\mathrm{PBu}_{3}^{\mathrm{t}}\right)\right](\mu-\mathrm{H})_{2}, \boldsymbol{2}$ showing $40 \%$ probability thermal ellipsoids. Selected interatomic distances $(\AA)$ are: $\operatorname{Pt}(1)-\operatorname{Ru}(4)=$ 2.8336(3), $\operatorname{Pt}(1)-\operatorname{Ru}(2)=2.8497(3), \operatorname{Pt}(1)-\operatorname{Ru}(3)=2.9969(3), \operatorname{Pt}(1)-\operatorname{Ru}(1)=3.1189$ (3), $\mathrm{Pt}(1)-\mathrm{H}(1)=1.67(4), \operatorname{Pt}(1)-\mathrm{H}(2)=1.71(4), \operatorname{Pt}(1)-\mathrm{P}(1)=2.3248(9)$. 


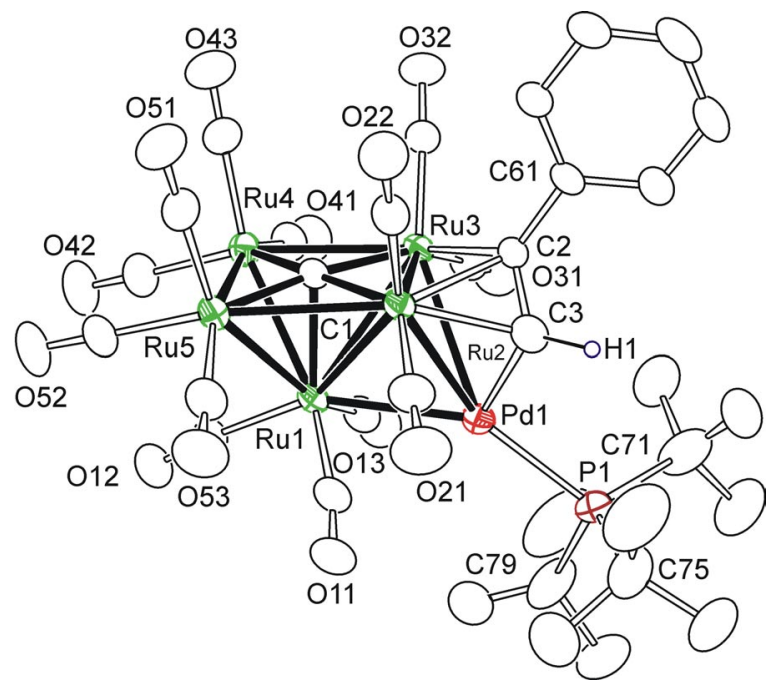

Figure 2. An ORTEP diagram of $\mathrm{Ru}_{5}(\mathrm{CO})_{13}\left(\mu_{5}-\mathrm{C}\right)\left(\mathrm{PhC}_{2} \mathrm{H}\right)\left[\mathrm{Pt}\left(\mathrm{PBu}_{3}^{\mathrm{t}}\right)\right]$, 3. Selected interatomic distances $(\AA)$ are: $\mathrm{Pt}(1)-\mathrm{C}(3)=1.980(7), \operatorname{Pt}(1)-\mathrm{P}(1)=2.3122(19), \operatorname{Pt}(1)-\operatorname{Ru}(1)$ $=2.6787(6), \operatorname{Pt}(1)-\operatorname{Ru}(2)=2.8476(6), \operatorname{Pt}(1)-\operatorname{Ru}(3)=2.9979(5), \operatorname{Ru}(1)-\operatorname{Ru}(5)=2.7705(8)$, $\mathrm{Ru}(1)-\mathrm{Ru}(4)=2.7949(8), \mathrm{Ru}(1)-\mathrm{Ru}(2)=2.9856(7), \mathrm{Ru}(1)-\mathrm{Ru}(3)=3.0018(7), \mathrm{Ru}(2)-$ $\mathrm{Ru}(3)=2.6666(7), \mathrm{Ru}(2)-\mathrm{Ru}(5)=2.8926(7), \mathrm{Ru}(3)-\mathrm{Ru}(4)=2.9037(7), \mathrm{Ru}(4)-\mathrm{Ru}(5)=$ $2.8898(8), \mathrm{Ru}(2)-\mathrm{C}(3)=2.210(7), \mathrm{Ru}(2)-\mathrm{C}(2)=2.214(6), \mathrm{C}(2)-\mathrm{C}(3)=1.369(10)$. 


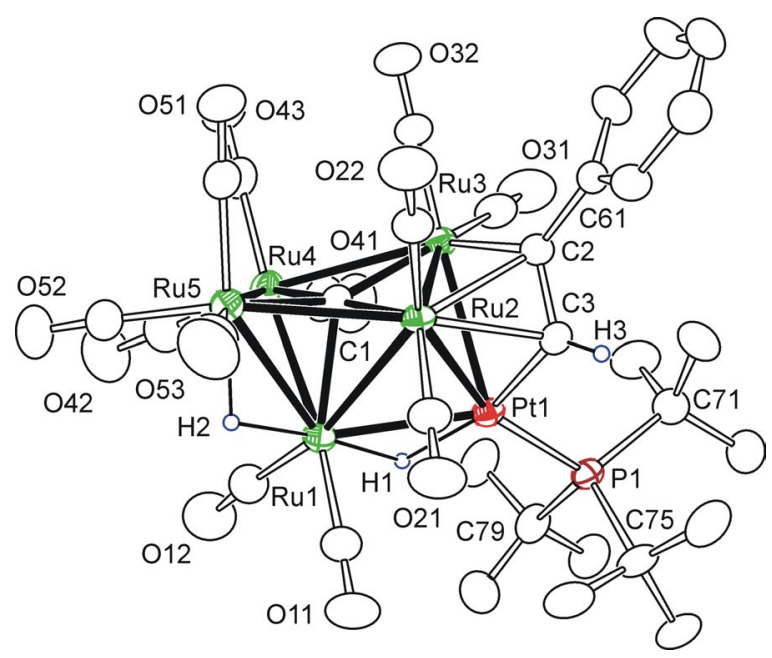

Figure 3. An ORTEP diagram of $\mathrm{Ru}_{5}(\mathrm{CO})_{12}\left(\mu_{5}-\mathrm{C}\right)\left[\mathrm{PtPBu}_{3}^{\mathrm{t}}\right]\left(\mathrm{PhC}_{2} \mathrm{H}\right)(\mu-\mathrm{H})_{2}, 4$ showing $40 \%$ probability thermal ellipsoids. Selected interatomic distances $(\AA)$ are: $\operatorname{Pt}(1)-\mathrm{C}(3)=$ 1.991(4), $\mathrm{Pt}(1)-\mathrm{P}(1)=2.3146(11), \mathrm{Pt}(1)-\mathrm{Ru}(1)=2.6793(4), \mathrm{Pt}(1)-\mathrm{Ru}(2)=2.7045(4)$, $\mathrm{Pt}(1)-\mathrm{Ru}(3)=2.8143(4), \mathrm{Pt}(1)-\mathrm{H}(1)=1.79(5), \mathrm{Ru}(1)-\mathrm{Ru}(5)=2.8161(5), \mathrm{Ru}(1)-\mathrm{Ru}(4)=$ 2.8609(5), $\mathrm{Ru}(1)-\mathrm{Ru}(2)=2.9208(5), \mathrm{Ru}(1)-\mathrm{Ru}(3)=3.6574(5), \mathrm{Ru}(1)-\mathrm{H}(1)=1.79(5)$, $\mathrm{Ru}(1)-\mathrm{H}(2)=1.73(5), \mathrm{Ru}(2)-\mathrm{C}(3)=2.230(4), \mathrm{Ru}(2)-\mathrm{C}(2)=2.289(4), \mathrm{Ru}(2)-\mathrm{Ru}(3)=$ 2.6694(5), $\mathrm{Ru}(2)-\mathrm{Ru}(5)=2.8906(5), \mathrm{Ru}(3)-\mathrm{C}(2)=2.050(4), \mathrm{Ru}(3)-\mathrm{Ru}(4)=2.9747(6)$, $\mathrm{Ru}(4)-\mathrm{Ru}(5)=2.8939(6), \mathrm{Ru}(5)-\mathrm{H}(2)=1.79(5) . \mathrm{C}(2)-\mathrm{C}(3)=1.395(5)$. 
Table 1. Crystallographic Data for Compounds 2, 3 and 4.

\begin{tabular}{|c|c|c|c|}
\hline & 2 & 3 & 4 \\
\hline Empirical formula & $\begin{array}{l}\mathrm{PtRu}_{5} \mathrm{PO}_{14} \mathrm{C}_{27} \mathrm{H}_{29} \\
\cdot 1 / 2 \mathrm{Et}_{2} \mathrm{O}\end{array}$ & $\begin{array}{l}\mathrm{PtRu}_{5} \mathrm{PO}_{13} \mathrm{C}_{34} \mathrm{H}_{33} \\
\cdot 1 / 2 \mathrm{C}_{8} \mathrm{H}_{18}\end{array}$ & $\begin{array}{l}\mathrm{PtRu}_{5} \mathrm{PO}_{12} \mathrm{C}_{33} \mathrm{H}_{35} \\
\cdot 1 / 2 \mathrm{C}_{8} \mathrm{H}_{18} \cdot{ }^{1 / 2} \mathrm{C}_{6} \mathrm{H}_{6}\end{array}$ \\
\hline Formula weight & 1345.97 & 1438.13 & 1451.19 \\
\hline Crystal system & Orthorhombic & Triclinic & Triclinic \\
\hline \multicolumn{4}{|l|}{ Lattice parameters } \\
\hline $\mathrm{a}(\AA)$ & $20.5414(9)$ & $12.6218(5)$ & 12.3991(9) \\
\hline $\mathrm{b}(\AA)$ & $21.3611(9)$ & $13.3776(5)$ & $12.6807(10)$ \\
\hline c $(\AA)$ & $18.4550(8)$ & $14.3656(5)$ & $16.5292(12)$ \\
\hline$\alpha(\operatorname{deg})$ & 90 & $92.678(1)$ & $105.157(2)$ \\
\hline$\beta(\mathrm{deg})$ & 90 & $91.051(1)$ & $104.028(2)$ \\
\hline$\gamma(\mathrm{deg})$ & 90 & $109.958(1)$ & $94.753(2)$ \\
\hline $\mathrm{V}\left(\AA^{3}\right)$ & $8097.8(6)$ & $2275.91(15)$ & $2403.8(3)$ \\
\hline Space group & Pccn & $\mathrm{P}-1$ & $\mathrm{P}-1$ \\
\hline $\mathrm{Z}$ value & 8 & 2 & 2 \\
\hline$\rho_{\text {calc }}\left(\mathrm{g} / \mathrm{cm}^{3}\right)$ & 2.208 & 2.099 & 2.005 \\
\hline$\mu(\operatorname{Mo~K\alpha })\left(\mathrm{mm}^{-1}\right)$ & 5.358 & 4.771 & 4.517 \\
\hline Temperature (K) & 296 & 296 & 296 \\
\hline $2 \Theta_{\max }\left({ }^{\circ}\right)$ & 56.6 & 56.6 & 56.6 \\
\hline 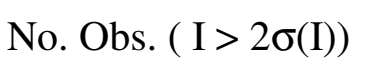 & 8493 & 9150 & 9934 \\
\hline No. Parameters & 474 & 515 & 554 \\
\hline Goodness of fit & 1.067 & 1.037 & 1.017 \\
\hline Max. shift in cycle & 0.002 & 0.001 & 0.001 \\
\hline Residuals*: R1; wR2 & $0.0241 ; 0.0556$ & $0.0454 ; 0.1139$ & $0.0335 ; 0.0741$ \\
\hline Absorption & SADABS & SADABS & SADABS \\
\hline Correction, Max/min & $1.000 / 0.549$ & $1.000 / 0.794$ & $1.000 / 0.698$ \\
\hline Largest peak in Final & 0.925 & 5.424 & 1.523 \\
\hline Diff. Map $\left(\mathrm{e}^{-} / \AA^{3}\right)$ & & & \\
\hline
\end{tabular}

